Philosophy and Progress: Vols. LI-LII, January-June, July-December, 2012 ISSN 1607-2278 (Print), DOI : http://dx.doi.org/10.3329/pp.v51i1-2.17678

\section{INVESTIGATING CRITERION FOR THEORY-CHOICE IN SCIENCE}

\author{
Md. Abdul Mannan*
}

\begin{abstract}
In science theory changes, the old is rejected and the new accepted, the new is considered to be better than the old, this betterment is the essence of scientific progress. But what is the criterion to measure one is better than the other? According to the very traditional view, it is 'fact' which determines the fate of a theory. But since fact itself is not theory free, it cannot be criterion for theory-choice. We find that theory replaces theory. In such a situation, what it is for which one theory can supersede the other. In this essay we have made an investigation about it and we finally find different criteria where in turn one criterion is found to be better than the other to explain scientific change.
\end{abstract}

\footnotetext{
* Associate Professor, Department of philosophy, University of Chittagong E-mail : mannan_philosophy@yahoo.com
}

\section{Facts determine theory-choice?}

It is a very regular phenomenon in the history of science that two (or more) competing theories claim truth about the same event in the natural world. In such an occasion scientists are to make their choice about which of the theories they are justified to hold on. Then they have to make a comparison between theories and choose the better one. So, they must have a rationality or criterion on which such a decision could be taken. Now, the objective of our investigation is what that criterion is on which scientists make their choice from those competing theories.

It is widely admitted that a theory is a human creation and the fact is given. That is, facts represent the reality. There exist at least some pure facts which are nothing but reality--fact is quite distinct from theoretical construct. So, when we justify acceptance of a theory we see whether there is agreement between the fact and the theory. If fact agrees, the theory is accepted; if not it is rejected. This is a very naïve understanding of the relation between theory and fact. In view of the above, we can say that fact is the arbiter for theorychoice. That is, fact will decide which of the competing theories should be accepted.

But philosophical investigation reveals that such a status of fact cannot sustain. Feyerabend holds that theories may be removed because of conflicting observations; and observations may also be removed for theoretical reason. This is because, he says, learning does not go merely from observation to theory but always involves both elements. Experience arises together with theoretical assumptions, not before them. For him, if we eliminate parts of the theoretical knowledge of a sensing subject, we have a person who is completely disoriented and 
incapable of carrying out the simplest action. Even, as to children's learning, the whole process starts only because the child reacts correctly towards signals, interpret them correctly. For, he processes the means of interpretation before he has experienced his first clear sensation. (Feyerabend, 168).

The greatest advances in science have not been accomplished by means of laws referring to observations, but rather by means of laws that speak of various hypothetical entities. Electric, magnetic and gravitational fields are among such hypothetical entities; they are not directly observable, and therefore, they are not theory independent. So they are metaphysical and speculative. The language representing the attributes of such hypothetical entities becomes theorydependent. (Shapere, 96) After the proved impossibility of Maxwell's demon and Heisenburg's principle of indeterminacy, men came to understand that the measuring instrument (man) is not simply a passive extension of our senses. Brody points out that an individual hypothesis can never be tested in isolation of a whole group of other theories. So, facts do not have power to make scientists accept the theory and reject it as well. (Brody, 96)

There is no pure observation which can be entitled to be the crucial observation for theory choice. The same thing is observed as different, 'seeing' is not just having the visual experience; it is also the way in which the visual experience is had. The physicists and the laymen see the same thing, but they do not make the same statement about it. The infant and the layman see; they are not blind; but they cannot see what the physicist sees. (Hanson, 15-17).

So, facts cannot determine theory-choice.

\section{Criterion in Falsification}

According to Popper, science starts from myth-making; and it becomes science when it faces a severe criticism. Here lies the question of criterion that on what reason we will accept or reject one mythical construct rather than other. For him, a myth is science when it is falsifiable; a scientific theory is accepted (or survive) when it is not refuted or falsified. This view of theory-choice is called theory of falsification. What is the exact nature of falsification? The above discussion about fact-theory relation reveals that facts alone cannot justify theory, because there is no such theory-independent pure fact which can judge between the theories neutrally. Then how can a theory-laden fact serve for justification? Popper, in this regards, holds that every test of theory whether resulting in its corroboration or falsification, must stop at some basic statement or other, which we decide to accept. If we do not come to any decision, and we do not accept some basic statement, then the test will lead us nowhere. The situation is never such that it compels us to stop at some particular basic statement, or else give up the test altogether. For any basic statement can in turn be subject to tests. This procedure does not have any natural end. Thus, if test is to lead us anywhere, we have to stop at some point and say that we are satisfied--though for the time being. (Popper, $1959,104)$

We do not make the test for the truth of the basic statement. In this sense it is conventional also, for it the statement which is taken for granted on the basis of agreement. They are accepted as result of decision to stop at. These accepted basic propositions are the basis for the corroboration of hypothesis, and thus indirectly for falsification. Experience can motivate a decision, that is, the acceptance or rejection of a theory, but a basic statement cannot be justified by them-no more by the thumbing of the table. (Bunge, 37). 
However, given the nature of basic statement, corroboration of a theory by any test premised on basic statements would be tentative; for we do know that this test is not the only test. There may be many other tests that may lead to falsification of the theory. But falsification of the theory would be final; for we know that at least this very test falsifies the theory. Attempt to falsify a theory is not a particular occasion, but a theory faces such an attempt every time. So, a theory is accepted until it is not refuted. A critical attitude towards a theory is a continuous event; a theory is always on trial to point at the error in it. Therefore, this method is also called the method of 'trial and error'. Popper says that in this method we first boldly propose a theory, then try our best to point out its possible errors. If our critical efforts do not succeed then we accept them tentatively. From this point of view, all laws, all theories are essentially tentative or conjectural or hypothetical even when we feel unable to doubt them any longer. For, before a theory has been refuted we can never know in what way it may have to be modified. (Popper, 1965,51).

Another aspect of this method is that only observation and experiment cannot decide upon the acceptance and rejection of a theory. For, there are many historical examples when a theory has not been rejected in the face of contradiction with the experimental facts. Newton's theory had no complete experimental support; it appeared to be in disagreement with natural phenomena; yet it survived for centuries until Einstein's theory appeared. So, according to the method of 'trial and error', a theory can be rejected only by that experiment which at the same time corroborates the falsifying hypothesis. There must be two theories which are competitors to each other. One can be rejected only in place of the other. (Popper, 1965, 54). Theory is not rejected without any alternative. And any alternation does not mean that the newly accepted theory is true or probable true--the falsification is complete and conclusive, but the corroboration is never so. (Krajewski, 71). Truth is hard to come by out of mistakes; it needs both ingenuity in criticizing old theory and ingenuity in the imaginative invention of new theory. (Pitt, 44). But it seems beyond human understanding; for, the test of old theory is based on basic statement which is conventionally accepted on the one hand; on the other hand, the new theory comes out of conjectureswe are unable to overcome these two predicaments of our understanding. Popper claims that a theory may be fittest in pragmatic sense, but may not be so in the spirit of truth. There are many false theories which often serve well enough: most of the formulations used in engineering and navigation are known to be false, although they are the excellent approximation and easy to handle, and they are used with confidence by people who know them to be false. (Popper, 1965, 56)

Since corroboration of a theory is tentative, choice about theory will not end. Any time in future there may appear other theory which will try to falsify the prior corroborated theory. This falsification may lead to the rejection of the previously accepted theory, and acceptance of yet newer theory. In this way the theory-choice activity will go on infinitely. It is infinite because both our ignorance and 'trial and error' process are infinite. Science, for Popper, starts from conjectures; there can never be any structural limitation for conjectures; so, it opens up all kinds of possibilities for imagination. On the other hand, world is infinitely complex. So, our choice-making activity will run infinitely. (Popper, 1965, 216) The infinite process of 'trial and error' results into an advancement of science. But growth in this process is not the accumulation of observations but rather repeated overthrow of scientific theories and their replacement by better or more satisfactory 
ones. So it is not like a library, as more and more books accumulate so more and more knowledge accumulates. But scientific growth occurs through criticism; it grows by a method more revolutionary than accumulative - by a method which destroys, changes, and alters the whole things including its most important instruments, the language in which our myths and theories are formulated.

If there is competition of this sort between the existing and falsifying hypotheses, the competing theories become incomparable. How then will we understand the growth among competing theories? A rational and fruitful discussion is impossible unless the participants share a common framework of basic assumptions, or at least, they have agreed on such a framework for the purpose of discussion. A discussion may also be difficult if the frameworks have little in common, and it will be easier if there is the greater overlap between the frameworks. (Pitt, 36). In this regard, according to the method of falsification, the criticism starts not from the question: how can we establish or justify our theories? But it rather starts from the question: what are the consequences of our theories? Thus criticism consists in comparing the consequences of different theories and tries to find out which of the competing theories has the consequences that seem preferable to us. (Pitt, 59-60). So, growth depends on the more and more interesting and difficult questions. For the more new answers scientists are induced to think of. Consequently, the greater the gap holds between scientists, the greater their intellectual horizon is extended. Every theory-choice is to explain the old category by theory of greater depth. There is no problem of comparing theories, for theories tries to solve the same family of problems. Though in this method we do not and cannot justify a theory, we can have a criterion, the criterion of criticism. Popper claims that history of science, like the history of all human ideas, is a history of irresponsible dreams, of obstinacy and of errors. But it is science in which errors are systematically criticized and fairly often corrected. We do make mistakes, but by recourse to continuous criticism, we often learn from our mistakes. (Popper,1965, 217). Our theories are replaced by competing theories through a critical discussion and thereby we graduate to better theoretical positions.

In this criterion there are three requirements for a theory to be better than its competitors. One, the new theory should proceed for some simple, new, powerful and unifying ideas about some connection between hitherto unconnected facts or new theoretical entities. This requirement is called simplicity. Two, the new theory should be independently testable, that is, it must have new and testable consequences. It must lead to the prediction of phenomena which have not so far been observed. Three, the theory should pass some new and severe tests. (Popper, 1965, 242). These three things are the relative potential satisfactoriness of a theory. If a new theory satisfies these three requirements, then the new one is better than the old.

This criterion of relative potential satisfactoriness also includes the consideration of the content of a theory. Popper says that the informative content of the conjunction, $a b$, of any two statements, $a$ and $b$, will always be greater than, or at least equal to, that of any of its components. That is $\mathrm{CT}(\mathrm{a}) \leq \mathrm{CT}(\mathrm{ab}) \geq$ CT(b). The theories of Kepler and Galileo were unified and superseded by Newton's logically stronger and better testable theory. Fresnel's and Faraday's by Maxwell's. Newton's and Maxwell's in turn were unified and superseded by Einstein's. So, if new theory provides the conjunction of two old theories, then the new is better than the old theory. But in this context the new theory with greater content would have the lesser 
degree of probability. But this not a discredit for a theory, because it means the high degree of falsifiability. Science aims more content, therefore, less probability, not vice-versa. 'Today is Monday' has highest degree of probability, whereas 'electron exists' has less degree of probability. Only a highly testable or improbable theory is worth testing, and is actually satisfactory if it withstands severe tests.

This is the criterion for theory-choice according to Popper. This is the method of falsification through which old theory is rejected and the new is accepted.

\section{Paradigm: Metaphysics of Science}

In the theory of falsification, due to the relative potential satisfactoriness, one theory can falsify the other, and in this way the scientists make their choice between the competing theories. No doubt, this is an improvement over the idea that facts determine the theory-choice. But there are some places that face the questions, especially regarding the regulative potential satisfactoriness of a theory. Popper has mentioned 'simplicity', 'testability' and 'truth-content' to compare the relative potential satisfactoriness of competing theories. But Thomas Kuhn claims that all the potentialities such as 'simplicity' and 'truth-content' are theory dependent. Some truth- content, for example, may be recognized by one theory, but may not be so by other theory. So, this cannot make any judgment between these two theories. This very argument of Kuhn's is the implication of his concept of paradigm.

In science, all achievements are not of the same level. There are some such achievements that particular scientific community acknowledge as supplying the foundation for its further practice. These achievements expound the body of accepted theories and illustrate many or all of its successful applications with exemplary observation and experiments. Aristotle's Physica, Ptolemy's Almagest, Newton's Principia and Optics, Franklin's Electricity, Lavoisier's Chemistry, Lyell's Geology and many others works are achievements of this kind. So, paradigm is the way of looking at the world, broad quasi-metaphysical insights or hunches about how the phenomena in some domain should be explained. The more we articulate the paradigm, the more we know what it is. Paradigm is a metaphysical model that more or less comprises the scientific community's beliefs about nature.

Thomas Kuhn says that paradigm determines on what aspects of nature do scientists report, what determines their choice, what motivates the scientists to pursue that choice to a conclusion, etc. Paradigm has all these things for normal science--- it provides a vision for normal science. In the history of science we see that from Tycho Brahe to E.O. Lawrence, some scientists have acquired great reputations, not for any novelty of their observations, but for the precision, reliability and scope of the methods they developed for redetermination of a previously known sort of facts. (Kuhn, 26). So, different paradigms make different worlds for their adherents.

If we ask an eminent physicist and a chemist whether a single atom of helium is or is not molecule, both would answer without any hesitation, but their answers would not be the same. For the chemist, the atom of helium was a molecule because it behaves like one with respect to the kinetic theory of gas. For the physicist, on the other hand, the helium atom was not a molecule because it displays no molecular spectrum. Presumably, both men are talking of the same particle, but they are viewing it through their own research training and practices. Their experiences of problem-solving told them what a molecule must be. Their answers were different because their paradigms were different. 


\section{Theory-Choice as Conversion Experience}

Normal science is rule-governed enterprise which makes progress by articulating the paradigm. But, sometimes situation may be such that any articulation of paradigm fails to assimilate certain anomalous phenomena. They are neither anticipated by the paradigm nor can the paradigm provide such rules to absorb them. Awareness of such anomalies is a necessary precondition for emergence of the new paradigm. Quantum mechanics was born from a variety of difficulties surrounding blackbody radiation, specific heat, and the photoelectronic effect. The situation when awareness of anomalies lasts so long and penetrates so deep is called scientific crisis.

When a paradigm remains dominant, failure is attributed to the scientists, because dominance of the paradigm ensures that there must have some solution. But when a paradigm is under the crisis, failure goes to the paradigm, because that time theory that status. A failure that had previously been personal may then come to be seen as the failure of theory under test. (Watkins, 28) Kuhn says that this failure reflects not on the paradigm but on the man - the man committed to the paradigm. Then his colleagues see him as 'the carpenter who blames his tools'. (Kuhn, 80)

In this situation of scientific crisis, a new interpretation of nature first comes in the mind of one or a few individuals. It is they who first learn to see science and the world differently. They are men so young or new to the crisis ridden field where practice has committed them less deeply. Now question is how are they able to convert the entire profession or relevant professional subgroup to their way of seeing science and the world? What causes the group to abandon the old paradigm in favor of the new? Is there any criterion on which scientists make their choice between the theories? According to
Feyerabend, no such criterion can exist. For him, history generally and the history of revolution in particular, is always richer in content, more varied, more many-sided, more lively and subtler than even the best philosopher and the best methodologist can imagine. It demonstrates to us the complexity of human change and the unpredictable character of ultimate consequences of any given act or decision of man. In this condition it cannot be believed that the naïve and simple minded rules which methodologists take as their guide are capable of accounting for such a maze of interactions. (Feyerabend, 19).

What occurs in the history of science is neither a decline nor a rise is determined by standards, but simply change demanded by the adoption of a new paradigm. The choice between competing paradigms cannot be resolved by any criterion of normal science. For Kuhn, two scientific schools disagree about what is a problem and what is solution. They will inevitably talk through each other's paradigms. Each paradigm will be shown to satisfy criteria it dictates. Within the new paradigm, the old terms, concepts, and experiments fall into a new relationship one with the other. For example, what Ptolemy meant by 'earth' was a fixed position; correspondingly, innovation of Copernicus was simply to move the earth. Practicing in different worlds, the group scientists see different things when they look from the same point in the same direction. They see different things in different relations. That is why a law that cannot even be demonstrated to one group of scientists may occasionally seem intuitively obvious to another. (Kuhn, 150).

Therefore, testing is possible for puzzle-solving within the paradigms, but not possible for decision about paradigms. For paradigm testing, question arises which of the two actual and competing theories fits the facts better. History of science 
shows that we cannot answer this question by any sort of testing. Neither Priestley nor Lavoisier's theory, for example, agreed precisely with existing observations, yet few contemporary scientists hesitated in concluding that Lavoisier's theory provided the better fit of the two. Now the question is: how scientists conclude that the theory is better than the old without any recourse to criterion? Laudan describes the situation as the case of logical indetermination. Although methodological rules and standards do constrain and delimit to some degree a scientist's choice of options those rules and standards are never sufficient to compel or unequivocally warrant the choice of one paradigm over the other. (Laudan, 1996, 89).

For Feyerabend, there is no single rule, however plausible and however firmly grounded in epistemology, which is not violated at some time or other. They are not result of insufficient knowledge or of inattention, which might have been avoided. On the contrary, we see that they are necessary for progress. Quantum theory, to mention among others, occurred because some thinkers either decided not to be bound by certain obvious methodological rules or because they unwittingly broke them. (Feyerabend, 1975, 23). Laudan says that every scientist has different reasons for his theory preference to those of his co-workers. So, it is category mistake to ask, say, why physicists think Einstein's theory is better than Newton's; for, there must be as many different answers to that question as there are physicists. (Laudan, 1996, 91). So, the act of judgment that leads scientists to reject a previously accepted theory is always based upon more than a comparison of that theory with the world.

New theory is less competent. So, if it is to succeed, the only way is to resort to other means than arguments. It will have to be brought about by irrational means such as propaganda, emotion, ad hoc hypothesis, and appeal to prejudices of all kinds. We need these irrational means in order to uphold what is nothing but a blind faith until we have found the auxiliary science, the facts, and the arguments that turn the faith into sound knowledge. (Feyerabend, 154). Copernicus who invents a counter induction as opposed to Ptolemy's theory became successful leading to progress acted first simply on faith.

So, the man who embraces a new paradigm at early stage must have faith that the new paradigm will succeed with many large problems in future. A decision of this kind can only be made on faith. Kuhn says, for a revolution, there must be at least few scientists who feel that the new proposal is on the right track and sometimes only personal and inarticulate aesthetic considerations can do that.

So, transfer of allegiance from one paradigm to other is a conversion experience that cannot be performed on the basis of method or criterion.

\section{Progressing and degenerating rate of problem-solving}

Though in the history of science, violation of the rules and methods indicates non-existence criterion, there is scope to look into the situation and then it would be possible to find out some other kind of standard that can determine theory-choice. Imre Lakatos maintains that there is such a rationality which includes this historical fact of the violation of rules and methods. History of science tells us that scientists have thick skin. They do not abandon a theory merely because facts contradict it. They invent some rescue hypothesis to explain the anomalies; or if they cannot explain the anomalies, they ignore it and direct their attention to other problems. When Newton publishes his Principia, it could not properly explain even the motion of the moon; in fact lunar motion refuted Newton's. 
Not only that, the same thing happened to Einstein's relativity theory. Kaufman, a distinguished physicist, refuted Einstein's theory in the very year it was published. But scientists rejected neither Newton's nor Einstein's for those refutations. In spite of this fact, scientists are not irrational men. Blind commitment is not an intellectual virtue, it is an intellectual crime. (Lakatos, 1-4). How is it possible that scientists will violate rules and methods yet they will remain rational? This is solved in Lakatos' concept of 'research program'. For him, the typical descriptive unit of great scientific achievements is not an isolated theory, but rather a 'research program'.

Science is not simply trial and error. For, the proposition, say, 'all swan are white' may be falsified by the discovery of one swan, but such trivial trial and error does not rank as science. Newtonian science could not have been science if it was just a set of four conjectures - three laws of mechanics and the law of gravitation. But rather it has been science because these four laws constitute only the 'hard core' of the Newtonian program. And there is something more than 'hard core', which is called auxiliary hypotheses that make a vast protective belt to keep the hard core protected from refutation. So, research program is a set of theories which constitute the 'hard core' and 'auxiliary hypotheses'. It is not just a isolated theory. Lakatos points out, more importantly, the research program also has a heuristic, that is, powerful problem-solving machinery that digests anomalies and turns them into positive evidence. For instance, if any phenomenon, say, motion of planet goes against Newtonian theory Newtonian scientists check his conjectures concerning atmospheric refraction, propagation of light in magnetic storms, and hundreds of other conjectures which are all part of the Newtonian program, so that they can explain away the difficulty. Even they may imagine a hitherto unknown planet and conclude its position, mass and velocity in order to explain the anomaly. (Lakatos, 4).
Therefore, all theories are born refuted and die refuted. All theories are not equally good; some have more powerful heuristic capacity to explain refuting facts and some have less powerful heuristic capacity. But all theories predict the novel facts. Research program that does not discover any novel facts, or that stops to discover, is a degenerating research program. On the other hand, any research program that continues to discover novel facts is a progressive research program. If we have two rival research programs, where one progressing and other is degenerating, scientists tend to join the progressing program. This is the rationale of scientific revolutions. Lakatos says that it is a matter of intellectual honesty to keep the record of degenerating program public, but it is not dishonesty to stick to a degenerating program and try to turn it into a progressive one. This means that the theory does not change instantly. A program may take decades before that get off the ground and become empirically progressive. There may be criterion, but it cannot result into the rejection instantly. Unless there is no better theory, merely some isolated facts can never make any criticism effective. Program criticizes another program. The progressing program replaces the degenerating one, neither instantly nor irrationally. (Lakatos, 6). No experimental result can kill a theory in a full blow. For, research program has heuristic power to solve the anomalies.

Every research program is possessed of some degree of autonomy for which a research program ignores the counter evidence. Such autonomy of a program makes a rational ground to give it some time for progress. For, this is intellectual honesty that one should not reject a program just with appearing a counter instance against it, without giving it the time and chance to show its competence to solve that. This is the reason why a scientist violates sometimes the existing rules. There is reason to violate reason. 
According to autonomy of research program, we should be modest in our hopes for our own projects because rival program may turn out to have the last word. (Hacking, 133). So, there is no experiment which can instantly overthrow a research program. There is no such thing as crucial experiment. An experiment is seen as crucial only long after the event. When a research program suffers defeat and superseded by another one, then one may, only after long hindsight, call an experiment crucial. Moreover, temperament of scientists affects their decision. A rash scientist may claim that his experiment defeated a program, and part of the scientific community may even, rashly, accept his claim. But if a scientist in the defeated camp put forward, a few years later, a scientific explanation of the allegedly crucial experiment within (or consistent with) the allegedly defeated program, the honorific title of the experiment may be withdrawn and the crucial experiment may turn from a defeat into a new victory for program. There are many experiments in the eighteenth century which were widely accepted as crucial evidence against Galileo's law of free fall, and Newton's theory of gravitation. But in the nineteenth century those turn out to be erroneous in the light of relativity theory. (Lakatos, 86-87).

Therefore, counter evidence or counter theory cannot determine the theory-choice, but only a progressing research program can replace a degenerating research program. This is the criterion of theory choice which includes scientists' rational choice and the reason for the violation of reason.

\section{Success and Criterion}

Research program discussed previously successfully pointed out that scientific theory is not any isolated one, but a set of ideas which includes a series of theories. In spite of this success rational reconstruction carried out by research program could not consider some realities in the history of science. Among other one is the fact that conceptual or ideological factors do influence theory-choice. Two, theory is not rejected or accepted in its entirety - there is continuity when change occurs. These two realities of history of science are considered in Laudan's concept of 'research tradition'.

There are some theories that are much more general, much less easily testable, set of doctrines or assumptions. Kinetic theory of gas is such a theory, to mention one among others. This is not a single theory, but a whole spectrum of individual theories that are historically and conceptually related. This is a grand theory which is the primary tools for understanding. Larry Laudan calls this type of theories scientific 'research tradition'. To understand anything we need ontology and methodology about that thing; and it is research tradition which provides the same. It provides a set of guidelines for the development of specific theories. The whole function of research tradition is to provide us with the crucial tools we need for problem-solving, both empirical and conceptual. A successful research tradition with its corresponding theories leads to the adequate solution of an increasing range of empirical and conceptual problems. So, success of a research tradition depends on problem-solving performance, not upon the a-temporal rationality. Success or progress is readily understood, rationality is far more obscure. Therefore, success is the criterion for theory-choice, in other words, which is called by Laudan problem-solving effectiveness.

Laudan says that the over-all problem-solving effectiveness of a theory is determined by assessing the number and importance of the empirical problems which the theory solves, and reducing the number and importance of the anomalies and conceptual problems which they generate. Problem-solving effectiveness may also come about simply by 
an expansion or the domain or solved empirical probiems with all the other vectors remaining fixed. In such a case the replace of $\mathrm{T} 1$ by $\mathrm{T} 2$ is clearly progressive. Effectiveness can also result from a modification of a theory, which eliminates some troublesome anomalies, or which resolves some conceptual problems. (Laudan, 1978, 69). This is the reason why a theory with less ability to solve empirical problems is accepted due to its ability to resolve some conceptual problems. This kind of choice was considered as scientists' pigheadedness, but it is now explained as rational. At the same time, previous success of a theory was considered only with regards to empirical problems, now conceptual resolution is also considered as success. So, empirical and conceptual both have come under the course of scientific change. Any a-temporal criterion cannot exist in face of progress - conceptual change may imply the change in criterion. Both theory and criterion for theorychoice can change each other-nothing is fixed and atemporal. Therefore, Aristotle was not being irrational when he claimed, in the fourth century B.C., that science of physics should be subordinate to, and legitimated by, metaphysics.

There are the shared problems which are the basis for rational appraisal of the relative problem-solving effectiveness of competing research traditions. Therefore, uniformitarian or catastrophist, Neptunist or vudeanist all agree about one and the same problem in any geological theory which was problem of explaining how many uniform and distinct layers had been formed. According to Laudan, we simply ask whether a research tradition has solved the problems that it set for itself; we ask whether it generated any anomalies or conceptual problems in its own process; we ask whether in the course of time, it has expanded the domain of solved problems and minimized that of anomalies and conceptual problems. If we do all these, then we should be able to construct something l1ke a progressive ranking of all research traditions in a given time.

When a research tradition fails to solve any important problem, then it is common for partisans of the tradition that they explore what sort of minimal changes can be made in the deep level methodology and ontology of that tradition to eliminate the anomalies and conceptual problems. Sometimes scientists find it impossible to think with one or another assumption of that tradition with a view to eliminate its anomalies and conceptual problems. At this point, scientists may most likely abandon the tradition. Even at this stage, they can choose to solve the anomalies and conceptual problems by introducing one or more modifications in the core assumption of a tradition. This is the way they preserve the bulk of assumptions of a tradition. (Laudan, 98-99).

So, research tradition undergoes a natural evolution - an evolution which represents a change that is far from repudiation of former tradition and a creation of a new one. If the research tradition has undergone numerous evolutions in the course of time, there may be many discrepancies between the methodology and ontology of its earliest and its latest formulations. Then we may see no degree of similarity between earlier and later stages in development of a tradition, but at the same time, they remain the same entity.

The old tradition changes into the new tradition. They are different traditions. Yet since the new emerges from the old, in the course of time, there is continuity between every successive stage from the beginning to the end.

\section{Conclusion}

In this investigation, we first got 'facts' as the arbiter of theorychoice. Facts are objective and neutral, so theory must be tested 
by facts. But we see that fact itself is theory-dependent. So, factual correspondence cannot be the criterion of theorychoice. Popper's method of falsification is advancement in this regard. According to method of falsification, theory falsifies theory. If T1 Falsifies T2 it is rational to accept T1 in place of T2. But Kuhn realizes that scientists may adhere to a defeated theory, which is unexplained in the method of falsification. In this context he maintains that in the situation of scientific crisis, no scope exists for rational choice. So, some kind of psychology, such as scientists' ability and commitment becomes decisive about which of the paradigms is to be accepted or rejected. Lakatos accepts Kuhn's view that the old theories are always richer than the new, yet scientists may hold up the new. It may appear as violation of reason. But he holds that reason cannot be violated without reason. If the new theory is progressive in problem-solving activity in the face of degenerating condition in problem-solving of the old, then it is not irrational to adhere the new. So, 'progressive/degenerating problem-shift' is the criterion for theory choice.

But Laudan realizes another aspect of theory choice. He says that no theory is rejected in its entirety. A theory consists of different parts of it. Scientists always modify the concerning parts and solve the new problems. In this way, the form of the theory is not totally different from the old. So, there is continuity in change. He also realizes that not only empirical problems, but also conceptual problems are decisive for theory choice. So if a theory T1 solves any conceptual problem then, in spite of $\mathrm{T} 1$ and $\mathrm{T} 2$ being equal in empirical problem-solving, it is rational to accept $\mathrm{T} 1$ for conceptual reason. Therefore according to Laudan 'problem-solving effectiveness' is the criterion of theory choice.

\section{References}

Brody, B.A. Science: Men, Method, Goals: a Reader, N.Y.: W.A. Benjamin Inc., 1968.

Bunge, M. The Critical Approach to Science and Philosophy, London: The Free Press, 1964.

Feyerabend, P.K. Against Method, London: New Left Books, 1975.

Hacking, I. Scientific Revolution, Oxford: Oxford University Press, 1981.

Hanson, N.R. Patterns of Discovery, Cambridge: Cambridge University Press, 1958.

Krajewski, W. Correspondence Principle and the Growth of Science, Dordrecht: D. Reidel Publishing Company, 1977.

Kuhn, T.S. The Structure of Scientific Revolutions, Chicago: University of Chicago Press, 1970.

Lakatos, I. The Methodology of Scientific Research Programme, Cambridge: Cambridge University Press, 1984.

Laudan, L. Progress and its Problems, New Delhi: Ambica Publications, 1978.

Laudan, L. Beyond Positivism and Relativism, Oxford: Westview Press, 1996.

Pitt, J. Rational Changes in Science, Dordrecht: D. Reidel Publishing Company, 1987.

Popper, K.R. The Logic of Scientific Discovery, London: Hutchinson and Com., 1959.

Shapere, D. Philosophical Problem of Natural Science, New York: The Macmillan Company, 1965.

Watkins, J.W.N. 'Against Normal Science', in Criticism and the Growth of Science, Lakatos, I. and Musgrave (Ed.), Cambridge: Cambridge University Press, 1965. 